\title{
Research of DOA Estimation Based on MUSIC Algorithm
}

\section{Liwei HUANG ${ }^{1, a}$ Huiqin $\mathrm{CHEN}^{2, b}$ Yulin $\mathrm{CHEN}^{3, \mathrm{c}}$ Haiyan $\mathrm{XIN}^{4, \mathrm{~d}}$}

${ }^{1,2,3,4}$ Department of Electrical Engineering, Chengxian college of Southeast University, Nanjing, 210088, China

\author{
aemail:liwei8203@163.com, bemail: 41024257@qq.com, \\ cemail: 4817382@qq.com, demail: 983511889@qq.com
}

Keywords: MUSIC Algorithm; DOA; Direction of Arrival

\begin{abstract}
This paper studies MUSIC algorithm in direction of arrival(DOA) estimation of signals, expounds the principle of this algorithm, conducts simulation for uniform linear array with Matlab, concludes that MUSIC algorithm has different performances in estimating direction of arrival with different number of arrays, different array element spacing or different SNR. The algorithm has high resolution, estimation precision and stability.
\end{abstract}

\section{Introduction}

Array signal processing is an important branch of signal processing, direction of arrival(DOA) estimation is an important part of array signal. MUSIC algorithm is used in DOA estimation. ${ }^{\text {[1] }}$ MUSIC algorithm decomposes the covariance matrix of received signal, promotes the rise of structure characteristics classification algorithm, has a good performance in DOA estimation and becomes symbol algorithm of spatial spectrum estimation theory. ${ }^{[2]}$ This paper compares and simulates with Matlab the characteristics of MUSIC algorithm in different conditions.

\section{MUSIC algorithm}

Suppose there are $\mathrm{M}$ uniform linear array, $\mathrm{N}$ incident narrowband source waves, the source direction is $\theta_{1} \quad \theta_{2} \ldots \theta_{N}, s_{i}(k)$ is the i-th complex amplitude of the source, $S(k)=\left[s_{1}(k), \cdots x_{N}(k)\right]^{T}$. Array direction vector is $a\left(\theta_{i}\right)=\left[1, e^{-j \omega_{i}}, \cdots e^{-j(M-1) \omega_{i}}\right]^{T} \quad(i=1 \ldots N)$, $A=\left[a\left(\theta_{1}\right), \cdots a\left(\theta_{N}\right)\right], \quad \omega_{i}=\frac{2 \pi d}{\lambda} \cdot \sin \left(\theta_{i}\right), d$ is the distance between array elements, $\lambda$ is the wavelength of carrier. ${ }^{[3]}$

According to the $\mathrm{N}$ received signals, get the estimation value of covariance matrix, decompose the eigenvalue of covariance matrix.

$$
R_{x}=E\left[X(k) X^{H}(k)\right]=A R_{s} A^{H}+\sigma^{2} I
$$

According to the order of the characteristic value, get $\mathrm{N}$ eigenvalues $\lambda_{1}, \lambda_{2} \cdots \lambda_{N}$ (by the order), take the number of eigenvalues and eigenvectors equal to the signal number for signal space, take the rest of the eigenvalues and eigenvectors for noise space, get the noise matrix. With the noise characteristic vector for columns, make the noise matrix

$$
E n=\left[v_{N+1}, v_{N+2} \cdots v_{M}\right]^{[4]}
$$

The eigenvectors correspond to noise eigenvalues and the column of matrix A correspond to source direction is orthogonal, so

$$
A^{H} v_{i}=0(\mathrm{I}=\mathrm{N}+1, \mathrm{~N}+2 \ldots \mathrm{M})
$$

Define the space spectrum

$$
\operatorname{Pmusic}(\theta)=\frac{1}{a^{H}(\theta) \operatorname{EnEn}^{H} a(\theta)}
$$

When the columns of $a(\theta)$ and $E n$ is orthogonal, the denominator is zero. Actually, because 
of the existed noise, the denominator is a small value, corresponds to the peak of Pmusic, the job is to find spectral peak to get the DOA estimation. ${ }^{[5]}$

\section{Simulation and comparison}

\section{DOA estimation performance of different number of array elements}

Experiment uses 3 separate narrowband signal source, the center frequency is $1000 \mathrm{~Hz}$, signal-to-noise ratio is 10, incident to the linear array from 60 degrees, 30 degrees, 45 degrees, the sampling number is 128 , take half wavelength as array element spacing. Take different numbers of array element of uniform linear array for simulation, the results are shown in figure 1.
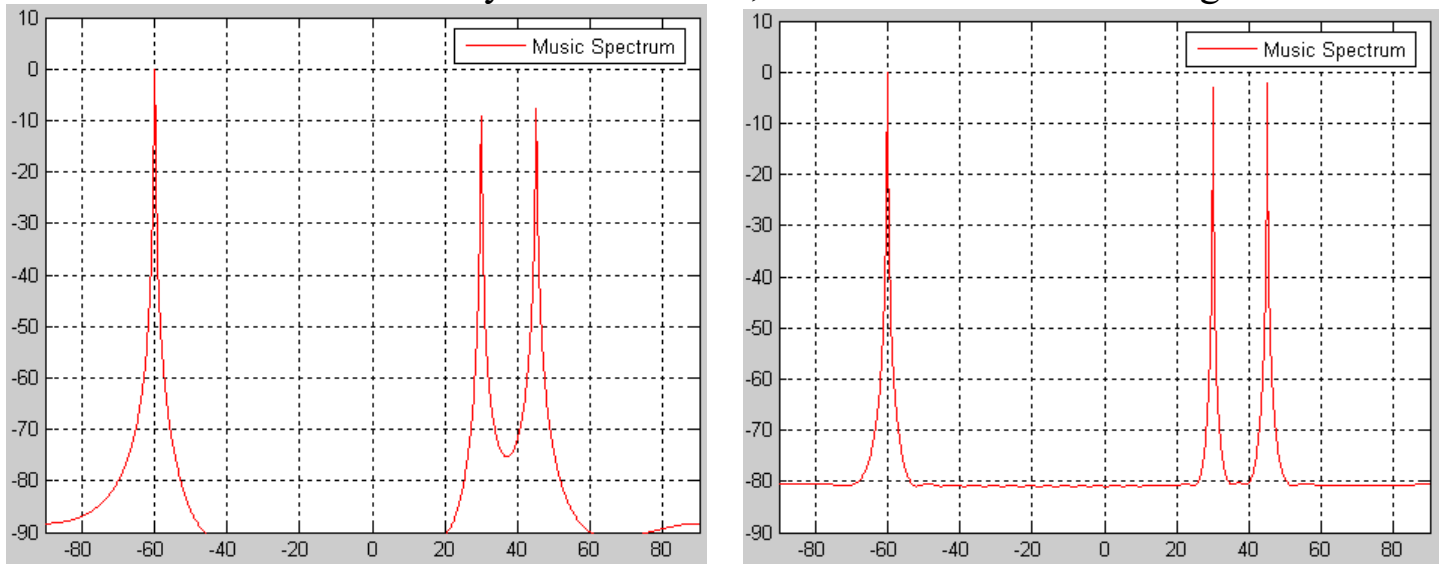

(a) number of array element is 10

(b) number of array element is 25

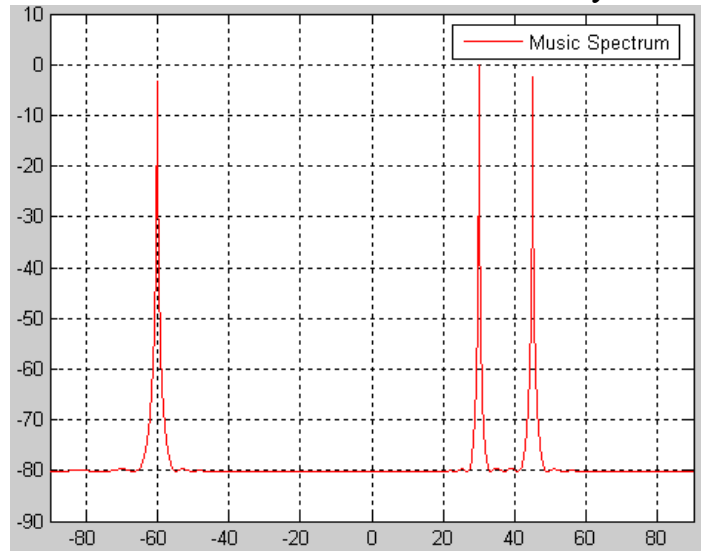

(c) number of array element is 40

Fig.1. Estimation performance of different number of array elements

Figure 1 shows that with the increase of distance between array elements, the resolution of MUSIC algorithm for DOA estimation has improved.

DOA estimation performance of different distances between array elements

Experiment uses 10 array element , 3 separate narrowband signal source, the center frequency is $1000 \mathrm{~Hz}$, signal-to-noise ratio is 10 , incident to the linear array from 60 degrees, 30 degrees, 45 degrees, the sampling number is 128 . Take different distances between array elements of uniform linear array for simulation, the results are shown in figure 2. 


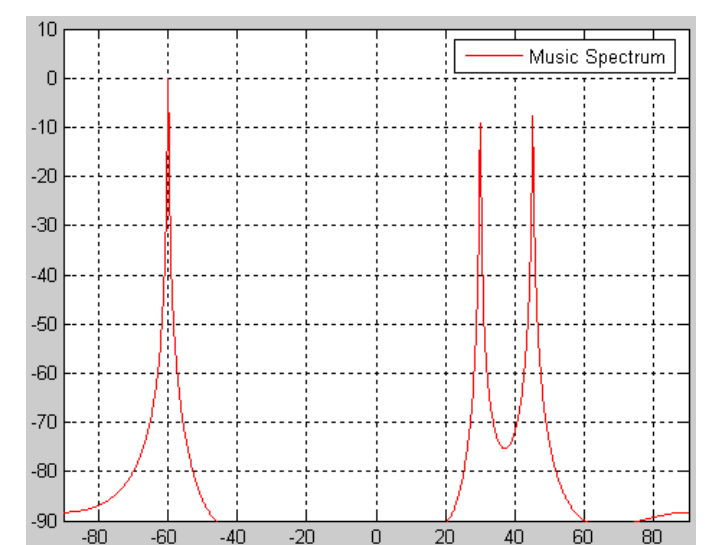

(a) half wavelength

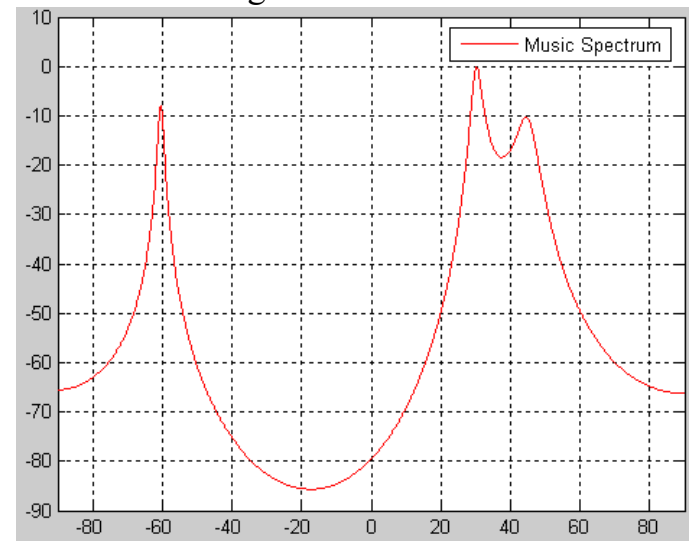

(c) one-eighth wavelength

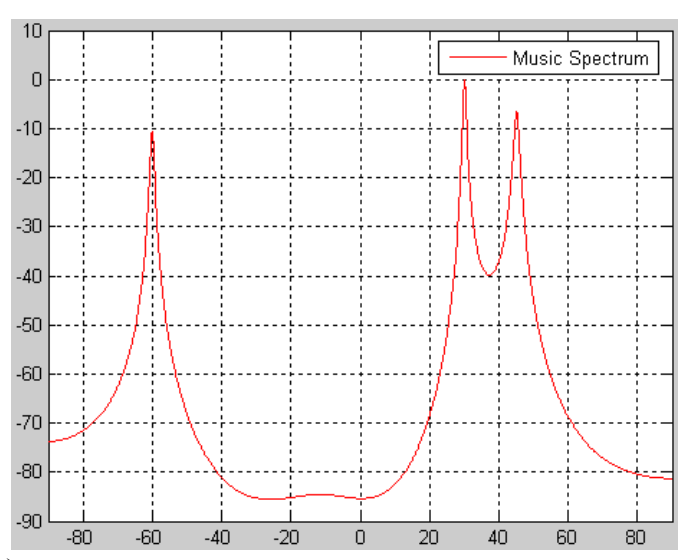

(b) quarter wavelength

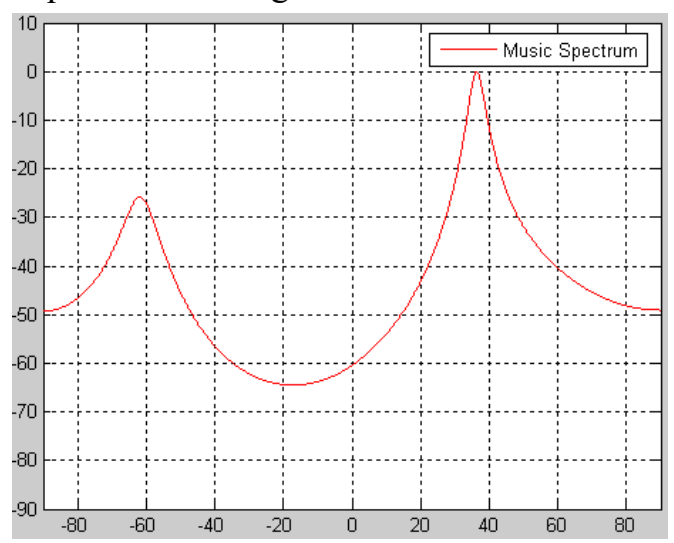

(d) one-sixteenth wavelength

Fig.2. Estimation performance of different distance between array elements

Figure 2 shows that with the increase of distance between array elements, the resolution of MUSIC algorithm for DOA estimation has improved.

DOA estimation performance of different signal-to-noise ratio

Experiment uses 10 array element, 3 separate narrowband signal source, the center frequency is $1000 \mathrm{~Hz}$, incident to the linear array from 60 degrees, 30 degrees, 45 degrees, the sampling number is 128 , take half wavelength as array element spacing. Take different signal-to-noise ratio of uniform linear array for simulation, the results are shown in figure 3.

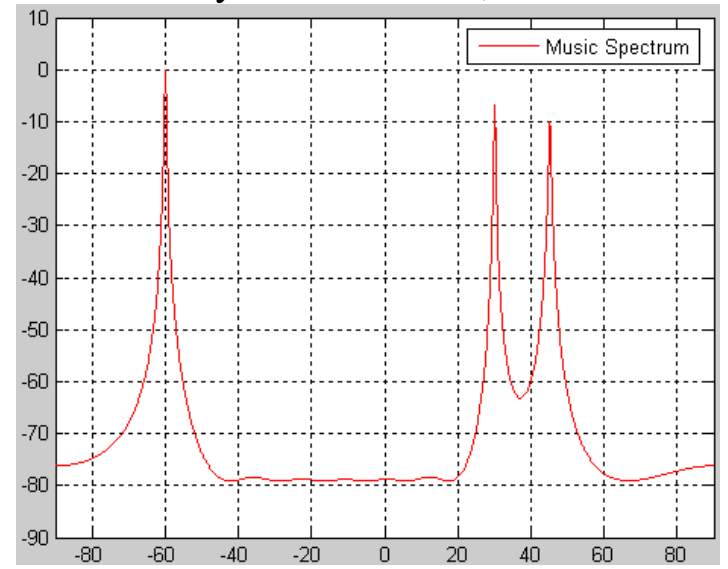

(a) SNR is 5

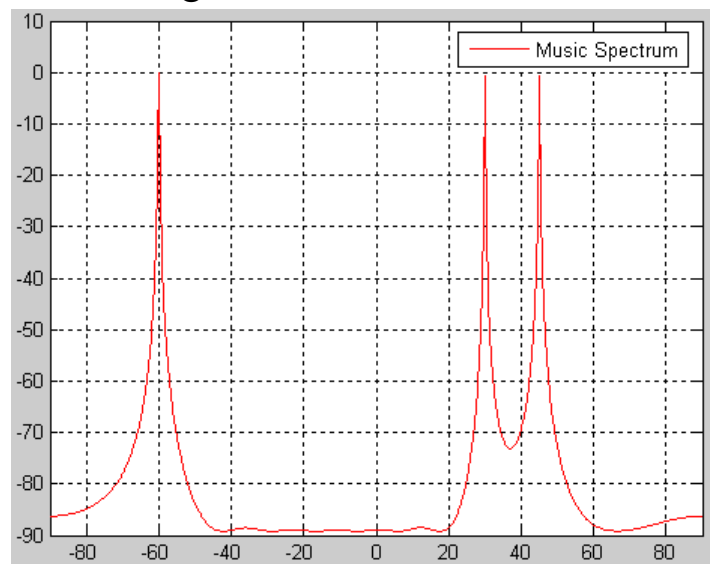

(b) SNR is 15 


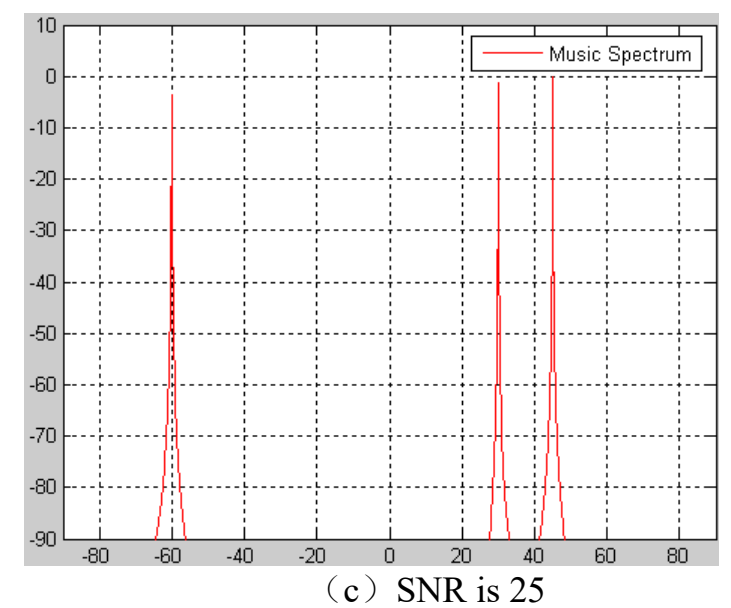

Fig.3. Estimation performance of different signal-to-noise ratio

Figure 3 shows that with the increase of signal-to-noise ratio, the resolution of MUSIC algorithm for DOA estimation has improved.

\section{Conclusion}

This paper analyzes the principle of MUSIC algorithm, simulates and compares the DOA estimation performance with different number of arrays, different array element space, different SNR. Experimental results show that with the increase of array element number, array element space, and signal-to-noise ratio, MUSIC algorithm for DOA estimation has higher resolution. Under certain conditions the algorithm has high estimation precision and stability.

\section{Acknowledgement}

In this paper, the research was sponsored by Philosophy and Social Science Foundation of Jiangsu Province (Project No. 2015SJD027) and Youth Fund Project of Chengxian college (Project No. y310001).

\section{References}

[1]T.J.Shan, M.Wax, T.Kailath. On spatial Smoothing for Direction-of-Arrival Estimation of Coherent Signals[J]. IEEE Transactions on Acoustic Speech and Signal Processing, 1985 33(4) 806-811.

[2]Jing min. Xin, Sano A. Linear prediction approach to direction estimation of cyclostationary signals in multipath environment[J].IEEE Trans.on Signal Processing, 2001 49(4)710-720.

[3]Schmidt R.O. Multiple emitter location and signal parameter estimation[J]. IEEE Trans. Antenna and Propagation, 1986 34(3) 276-280.

[4] A. Errikson, P. Stoica , T. Soderstorm. Markov-based eigenanalysis method for frequency estimation [J]. IEEE Trans. Signal Processiny, 1994 42(3) 586-594.

[5] Liu T H, Mendel J M. A subspace based direction finding algorithm using for fractional lower order statistics[J]. IEEE Trans Signal proeessing, 200149 1605-1613. 